Results NAFLD was diagnosed in $8.5 \%$ of overweight and $24.9 \%$ of obese children. In obese children the prevalence of NAFLD was $22.3 \%$ for age group 6-11.9 years and $35.5 \%$ for age group 12-19 years. There was no significant difference between girls and boys $(\mathrm{P}=0.521)$. Age was similar in children with and without NAFLD $(\mathrm{P}=0.766)$. An increase of $10 \mathrm{U} / \mathrm{L}$ of ALT, AST and ALK increased the odds of NAFLD $6 \%, 5 \%$ and $3 \%$, respectively. An increase of $10 \mathrm{mg} / \mathrm{dl}$ of triglycerides and glucose were associated with a $12 \%$ and $8 \%$ increase and one of TSH with a 15\% increase in the odds of NAFLD. An increase of 1 unit of HOMA-IR was associated with a $21 \%$ increase in the odds of NAFLD.

Conclusions ALT, AST, ALK and HOMA-IR can predict the progression of NAFLD. Findings emphasize on the importance of prevention of obesity and early intervention to prevent abnormalities among obese children.

\section{OC50 ACUTE LIVER FAILURE IN CHILDREN: AETIOLOGY AND EVOLUTION}

\footnotetext{
1,2Alina Grama, ${ }^{2}$ Lucia Burac, ${ }^{1,2}$ Simona S Cainap, ${ }^{2}$ Cornel Aldea, ${ }^{2}$ Dan Delean, ${ }^{2}$ Bogdan Bulata, ${ }^{2}$ Diana Pacurar, ${ }^{2}$ Laura Bodea, ${ }^{2}$ Claudia Sirbe, ${ }^{1,2}$ Tudor L Pop*. ${ }^{1}$ University of Medicine and Pharmacy 'Iuliu Hatieganu' Cluj-Napoca, Romania, Cluj-Napoca, Romania; ${ }^{2}$ Emergency Clinical Hospital for Children, Cluj-Napoca, Romania
}

10.1136/archdischild-2019-epa.47

Introduction Acute liver failure (ALF) is a syndrome defined by rapid decline of hepatic function, jaundice, coagulopathy (INR >1.5) and hepatic encephalopathy in patients with no evidence of previous liver disease. The aetiology of ALF in children varies on the age.

Material and methods We have analysed retrospectively the aetiology of the ALF in children followed-up in our clinic during 7-year period (January 2012 - December 2018). We studied the main causes of ALF based on the age of children and their evolution after treatment.

Results During this period, 97 patients (46 males, 47.42\%) were admitted with ALF: 33 infants (0-12 months), 30 children (1-14 years) and 34 adolescents (14-18 years).

In infants the most important causes of ALF were viral and bacterial infections: 24 patients (72.72\%). Cytomegalovirus, Epstein-Barr virus, Herpesvirus, Parvovirus or Gram-negative bacteria were involved, with a high mortality: 10 patients (30.30\%). Metabolic disorders were the cause of ALF in 9 patients (43.47\%): 3 infants with mitochondrial disorder, 2 infants with galactosemia and tyrosinemia respectively, and one with neonatal haemochromatosis and hereditary fructose intolerance. Of those, 3 survived after supportive therapy and diet, one is alive after liver transplantation and 5 died.

In children, toxic hepatitis was the most important cause of ALF (18 patients, 60\%): albendazole in 11 children, mushrooms in 3 children, and one child with each acetaminophen, valproate or fluconazole. Viral and bacterial infections were the causes of ALF in 5 children (16.66\%), 2 of them developed fatal aplastic anaemia. There were also 4 children with autoimmune hepatitis (AIH, 13.33\%) and 3 children with Wilson disease (WD, 10\%) presenting ALF at the time of diagnosis. The mortality in children with ALF was $20 \%$ (6 patients).

Drugs and mushrooms intoxication was the most common cause of ALF among adolescents: 27 patients (79.41\%), acetaminophen being the most often involved (17 patients). Fortunately, the mortality was low and only one patient died after colchicine overdose. There were 4 patients with $\mathrm{AIH}$ and 4 with WD. One patient died with Fulminant WD, other 2 were transplanted and the others are alive with supportive therapy.

Conclusions In children, the aetiology of ALF is different depending on age and is associated with a high mortality despite optimal medical therapy, but without emergency liver transplantation. The management of this cases requires a multidisciplinary team involving paediatric hepatologist, critical care specialist, nephrologist or liver transplant surgeon.

\section{OC51 ANTI-PARIETAL CELL ANTIBODIES IN CHILDREN WITH CELIAC DISEASE. GLUTEN FREE DIET EFFECT}

1,2 Valeria Novicova, ${ }^{1}$ Natalia Shapovalova*, ${ }^{1}$ Maria Revnova, ${ }^{3}$ Elena Kalinina, ${ }^{2}$ Sergei Lapin, ${ }^{1}$ Olga Gurina, ${ }^{1}$ Elena Dementieva, ${ }^{1}$ Ruslan Nasyrov. ${ }^{1}$ St. Petersburg State Pediatric Medical University, St. Petersburg, Russian Federation; ${ }^{2}$ St. Petersburg First Medical University, St. Petersburg, Russian Federation; ${ }^{3}$ Mechnikov I.I. North-Western State Medical, St. Petersburg, Russian Federation

\subsection{6/archdischild-2019-epa.48}

Aim To evaluate the prevalence of anti-parietal cell antibodies (APCA) in children with celiac disease(CD) and gluten free diet (GFD) effect

Methods The first group consisted of 58 children with chronic gastritis (CG) and newly diagnosed CD who didn't adhere to the GFD, the second group included 49 children with CG and CD, who were on GFD. The comparison group 3 consisted of 69 children with CG and excluded CD. To confirm or exclude $\mathrm{CD}$, according to the recommendations of ESPHGAN, the serological examination (anti-tTG IgG, IgA and anti-DPG IgG by ELISA), morphological study, were carry out for all patients. And 80 patients underwent genetic testing to determine the HLA-DQ2/HLA-DQ8 genes using PCR.

All patients underwent a same examination: histological study of gastric biopsies, histological verification of $\mathrm{H}$. pylori infection and biopsy urease test. Identification of APCA: antiIntrinsic Factor (ELISA) was carried out in 140 patient and anti- $\mathrm{H}+/ \mathrm{K}+$ ATPase (ELISA) in 58. APCA, using indirect immunofluorescence reaction, were determined in 45 children. Immunohistochemical determination of anti-tTG deposits in the stomach and duodenal mucous was performed on 10 patients.

Results The frequency of elevated levels of APCA was in group $1-10,00 \%$, in group $2-0 \%$, in group $3-5,43 \%$ $\left(\mathrm{P}_{1,2}=0,012 ; \mathrm{P}_{1,3}=0,492 ; \mathrm{P}_{2,3}=0,065\right)$. The prevalence of fundal gastric atrophy didn't differ statistically significant in groups, $p>0,05$ Correlation analysis didn't reveal morphometric duodenal mucosal parameters association with the gastric mucosa in group 1 and $3, p>0,05$. In group 2 a strong direct correlations between the depth of the fundal gastric pits and depth of the duodenal crypts $(r=0,818)$ and between number of parietal cells and intraepithelial lymphocytes in duodenum $(\mathrm{r}=-0,369) ; \mathrm{p}<0,05$ were found. Deposits of anti-tTG antibodies was detected in duodenal mucous and wasn't found in gastric mucous. APCA didn't correlate with anti-tTG antibodies, $\mathrm{p}>0,05$.

Conclusion The prevalence of APCA in children with CD (1:10) was higher than in children with isolated gastritis (1:20), but statistically not significant. However, the prevalence of APCA is less among patients on a GFD statistically significant. The possible protective effect of a GFD on APCA requires additional study. 